\title{
ATUAÇÃO TERAPÊUTICA OCUPACIONAL NA EQUOTERAPIA: UMA REVISÃO DE LITERATURA
}

\author{
ACTUACIÓN TERAPÉUTICA OCUPACIONAL EN LA EQUINOTERAPIA: UNA
} REVISIÓN DE LA LITERATURA

\section{OCCUPATIONAL THERAPEUTICS IN HIPPOTHERAPY: A LITERATURE REVISION}

\section{Letícia Nayara Gonçalves' ${ }^{1}$ Ana Carollyne Dantas de Lima ${ }^{2}$}

\begin{abstract}
RESUMO
Introdução: A Equoterapia é empregada como um método terapêutico que utiliza o cavalo com a finalidade de proporcionaro desenvolvimento global do praticante, o qual tem sua base nos conceitos da equitação e nas atividades realizadas por profissionais em conjunto com o animal. Seguindo essa linha, o terapeuta ocupacional tem competência para desenvolver práticas que oportunizem ao indivíduo maior nível de independência e autonomia em suas ocupações e melhor qualidade de vida. Objetivo: Desta forma, este estudo se propôs a levantar na literatura pesquisas que apresentem as ações e intervenções da Terapia Ocupacional na Equoterapia. Métodos: A pesquisa foi realizada nas plataformas de busca Pubmed e Bireme e nas bases de dados: SciELO, LILACS e MEDLINE. Foram utilizados, na língua portuguesa e inglesa, descritores presentes nas bases DeCS e MeSH e termos livres disponíveis. Foram incluídos 3 estudos considerados pertinentes e que corresponderam aos critérios de inclusão estabelecidos. Resultados: A partir da busca de descritores e termos livres foram identificados 194 artigos, os quais após verificação de elegibilidade resultaram em 3 artigos para análise nesse estudo. Conclusão: Os resultados permitiram afirmar que a interação entre homem e cavalo proporciona uma gama de benefícios, assim como a atuação do terapeuta ocupacional mostrou eficácia. No entanto, ainda é escasso o número de pesquisas que abordem a prática do terapeuta ocupacional na Equoterapia, assim necessitando de mais estudos acerca do tema.
\end{abstract}

\section{PALAVRAS-CHAVE}

Prática Profissional; Terapia Ocupacional; Terapia Assistida por cavalos.

\section{RESUMEN}

Introducción: La Equinoterapia es empleada como un método terapéutico que utiliza el caballo con la finalidad de proporcionar el desenvolvimiento total del practicante, lo cual tiene su base en los conceptos de la Equitación y en las actividades realizadas por profesionales en conjunto con el animal. Siguiendo esa línea, el terapeuta ocupacional tiene competencia para desarrollar prácticas que faciliten al individuo un mayor nivel de independencia y autonomía en sus ocupaciones y mejor calidad de vida. Objetivos: De esta forma, en este estudio se propuso levantar en la literatura búsquedas que presenten las acciones e intervenciones de la Terapia Ocupacional en la Equinoterapia. Metodología: La búsqueda fue realizada en la plataformas PubMed y Bireme y en las bases de datos, SciELO, LILACS y MEDLINE. Fueron seleccionados, en la lengua

1 Graduanda em Terapia Ocupacional pela Universidade Federal da Paraíba (UFPB). Email: leticianayara1416@gmail.com Endereço: Rua Osvaldo Cuz, 208, Bayeux - PB, 5830940. Telefone: 55 (83) 996162176. ORCID: https://orcid.org/0000-0002-7915-4963

2 Mestre em Saúde da Comunicação Humana pela Universidade Federal de Pernambuco (2013) e Doutora em Neuropsiquiatria e Ciências do Comportamento (UFPE). Possui formação básica em Equoterapia pela ANDE - BRASIL. Professora adjunta do Departamento de Terapia Ocupacional da Universidade Federal da Paraíba (UFPB). Email: ana_cdlima@yahoo.com.br Endereço: Rua Tab. Stanislau Eloy, 258-304 Castelo Branco, João Pessoa - PB, 58050-585. Telefone: (83) 3216-7996. ORCID: 0000-0002-2735 
portuguesa e inglesa, descritos en las DeCS, MeSHy términos libres disponibles. Fueron concluidos 3 estudios considerados pertinentes y que corresponden a los criterios de inclusión, establecidos. Resultados: A partir de la búsqueda de descriptores y términos libres se identificaron 194 artículos, los cuales después de verificación de elegibilidad resultaron en 3 artículos para análisis en ese estudio. Conclusiones: Los resultados permitirán afirmar que la interacción entre hombre y caballo proporciona una gama de beneficios, así como la actuación del terapeuta ocupacional mostró eficacia. Portanto, aún es escaso el número de búsquedas que aborden la práctica del terapeuta ocupacional en la Equinoterapia, necesitando de más estudios acerca del tema.

\title{
PALABRAS CLAVE
}

Practica; Terapia Ocupacional; Terapia Asistida por Caballos

\begin{abstract}
Introduction: The Hippotherapy is used as a therapeutic method that uses the horse with the purpose to provide the overall development of the practitioner; it is based on the concepts of riding and the activities performed by professionals in conjunction with the animal. Following this line, the occupational therapist is competent to develop practices that give the individual a higher level of independence and autonomy in their occupations and a better quality of life. Objective: In this way, this study proposed to lift in the literature the forms of intervention used by Occupational Therapy in Hippotherapy, as well as the practices performed in that intervention. Methods: The research was carried out on the Pubmed and Bireme search platforms and in the databases: SciELO, LILACS and MEDLINE. Descriptors were used in the databases DeCS and MeSH and free terms available in portuguese and English. Three studies considered relevant and that corresponded to the established inclusion criteria were included. Results: From the search for descriptors and free terms, 194 articles were identified, which after verification of eligibility resulted in 3 articles for analysis in this study. Conclusion: The results allow to affirm that the interaction between man and horse provides a range of benefits, just as the performance of the Occupational Therapist has shown to be effective. However, there is still little research that addresses the practice of occupational therapists in equine therapy.
\end{abstract}

\section{KEYWORDS}

Occupational Therapy; Equine-Assisted Therapy; Professional Practice

Recibido: 12 Noviembre 2018

Aceptado: 19 Noviembre 2019 


\section{INTRODUÇÃO}

O conceito da Equoterapia é formulado pela Associação Brasileira de Equoterapia (ANDE) (n.d.a) como um método terapêutico e educacional que através do emprego do cavalo buscar objetivar o desenvolvimento biopsicossocial de pessoas com deficiências seguindo uma abordagem interdisciplinar.

Os primeiros benefícios da prática equestre foram identificados na Grécia antiga, por Hipócrates (458-370 a.C.) e, somente a partir das décadas de 1960 e 1970 alguns países europeus e os Estados Unidos passaram a utilizar a técnica para o tratamento de paralisia cerebral, síndrome de Down e outras alterações neurológicas. No Brasil, a prática da Equoterapia adquiriu reconhecimento através da ANDE (n.d.b.) em 1989. Nesta perspectiva, a prática equestre, compreendendo o praticante dentro de uma perspectiva holística, tem como base científica o movimento tridimensional do passo do cavalo, a fim de promover ganhos de ordem física, psicológica, social e educacional.

O cavalo é naturalmente dotado por três tipos de andaduras: passo, trote e galope. Dentro da abordagem da Equoterapia o principal tipo de andadura utilizado é o passo, pois o movimento tridimensional acentuado possui semelhança com o movimento do corpo humano. Também conhecida como andadura natural ritmada, ela é compassada e executada em quatro tempos (Fontana, Monteiro, Fick, \& Zorzo, 2010).

A montaria na Equoterapia pode ser de dois tipos, individual e em dupla. A escolha será realizada através das limitações do praticante e dos objetivos traçados pelo profissional responsável. A montaria individual é indicada para praticantes que sejam aptos para ficarem sozinhos no cavalo, quando os objetivos dos atendimentos forem voltados para o desenvolvimento da independência e quando a conduta é direcionada para o fortalecimento muscular. Já a montaria em dupla é indicada para praticantes que possuem ou apresentam dificuldades em sua estabilidade corporal e que prejudique sua sustentação no cavalo, dessa forma ele é acompanhado por um terapeuta no dorso do cavalo (Luz, Boaretto \& Rodrigues, 2018).

Empregando as palavras de Citterio (1999), a Equoterapia pode ser classificada como uma ferramenta dotada por técnicas reeducativas que visam a estimulação e a potencialização dos aspectos sensoriais, motores, cognitivos e comportamentais utilizando o cavalo como artifício para a execução de atividades com caráter lúdico-esportiva.

Walter e Vendramini (200o) enfatizam que as técnicas de equitação e as atividades equestres proporcionadas ao praticante exigem a participação do corpo inteiro, proporcionando a conscientização do próprio corpo, bem como contribuições que alcançam o desenvolvimento do equilíbrio estático e dinâmico, regulação do tônus e da força muscular, melhora da coordenação motora, da atenção, da autoconfiança e na interação social. Assim como apresentado por Freire (1999), o praticante se transforma num participante ativo no processo de terapia, visto que o indivíduo está sujeito a vivenciar estímulos produzidos pelos movimentos realizados pelo cavalo na pelve do praticante.

Para Prestes, Weiss e Araújo (2010), tanto nas atividades individuais como nas em grupo, o praticante desenvolve o respeito e aprende a cuidar do outro. A interação com o terapeuta e, principalmente com o cavalo proporciona o conhecimento de que é necessário trabalhar em conjunto, cooperando com o próprio animal e com as pessoas em volta.

Os ganhos promovidos pela Equoterapia são mediados por profissionais que atuam em uma equipe multiprofissional em uma perspectiva interdisciplinar. A equipe mínima é composta por um adestrador de cavalo, um fisioterapeuta e um psicólogo. Porém, como afirma Silveira e Wibelinger (2010) também há necessidade de que outros profissionais façam parte desta equipe, dentre estes o terapeuta ocupacional.

Conforme o Conselho Regional de Fisioterapia e Terapia Ocupacional da $3^{\circ}$ região, (CREFITO 3), que corresponde ao estado de São Paulo, a Terapia Ocupacional é uma profissão da área da saúde que possui uma ampla formação nas Ciências que abordam a saúde, o social, o comportamento, os aspectos psicossociais e ocupacionais. Centrada no cliente, o terapeuta ocupacional possui competência para promover saúde e bem-estar através da ocupação. Trabalhando com pessoas e comunidades sua finalidade é ampliar as capacidades de inserção e participação nas atividades cotidianas que exerçam significado e importância nas vidas delas. 
O terapeuta ocupacional enxerga que um sujeito saudável é aquele capaz de estar envolvido ativamente no contexto em que está inserido. Dessa forma, os profissionais devem estar capacitados para visar e executar ações que fortaleçam e tornem possível o engajamento e a participação dos clientes em ocupações significativas para ele e que promovam saúde (Wilcock \& Townsend, 2014 como citado em Associação Americana de Terapia Ocupacional [AOTA], 2015).

No âmbito da Equoterapia, o objetivo do terapeuta ocupacional é proporcionar ao praticante o maior nível de independência e autonomia em suas ocupações por intermédio do cavalo. Segundo (AOTA) (2015), a Estrutura da Prática da profissão descreve que as ocupações são caracterizadas como atividades nas quais os indivíduos se engajam diariamente, podendo ser categorizadas como Atividades de Vida Diária (AVD), Instrumentais de Vida Diária (AIVD), educação, trabalho, brincar, lazer, descanso e sono e participação social.

Assim sendo, este profissional realiza uma avaliação inicial e traçar os atendimentos baseado em sua análise profissional, juntamente com os objetivos almejados pelo cliente (Gadelha, 2016). As atividades elaboradas e propostas precisam atender as necessidades individuais, os aspectos pessoais e o nível funcional do praticante. O terapeuta deverá atuar, primeiramente, buscando a construção de vínculo e, consecutivamente, propondo ações que incentivem a expressão corporal e emocional do praticante. Sendo assim, o terapeuta ocupacional na abordagem equoterápica, para alcançar seus objetivos terapêuticos, utiliza o cavalo como um instrumento facilitador proporcionando autoconfiança ao praticante por meio de atividades que preservem suas limitações e enfatizem suas possibilidades (Cavalcanti \& Calil, 2007).

Apesar de poder fazer parte da equipe que compõe a Equoterapia e do reconhecimento desta prática pelo conselho da profissão, a literatura da área não apresenta clara e diretamente a atuação do terapeuta ocupacional neste campo, e o mesmo ainda não é reconhecido como profissional da equipe mínima, o que demonstra a necessidade de produção de estudos que analisem e comprovem a relevância e os ganhos proporcionados pelo terapeuta ocupacional na Equoterapia e a necessidade do mesmo compor a equipe básica. Se faz necessário também o aumento das pesquisas científicas que envolvem terapeutas ocupacionais como forma de divulgar e evidenciar cientificamente o método terapêutico utilizado por este profissional. Desta forma, pretende-se analisar o estado de conhecimento atual e as evidências científicas sobre as ações e intervenções da Terapia Ocupacional na Equoterapia.

\section{MÉTOdo}

Trata-se de um estudo de levantamento bibliográfico por meio da realização de uma revisão integrativa. Este tipo de método foi escolhido pois permite a síntese de múltiplos estudos publicados e possibilita conclusões gerais a respeito de uma área particular de estudo (Mendes, Silveira \& Galvão, 2008).

Para a elaboração da presente revisão integrativa as seguintes etapas foram percorridas: formulação da pergunta condutora e objetivos da revisão integrativa; estabelecimento de critérios de inclusão e exclusão de artigos; definição das bases de dados e informações que foram extraídas dos artigos selecionados; apresentação e análise, e elaboração da discussão (Souza, Silva \& Carvalho, 2010).

Desta forma, a seguinte questão foi formulada para nortear esta revisão: Quais as ações e intervenções da Terapia Ocupacional na Equoterapia? O objetivo deste trabalho é analisar o estado de conhecimento atual e as evidências científicas sobre as ações e intervenções da Terapia Ocupacional na Equoterapia.

\section{Estratégias de busca}

A partir desse questionamento, os critérios de inclusão dos artigos foram: artigos publicados em português e inglês, que apresentaram as possibilidades de atuação da Terapia Ocupacional na Equoterapia com os resumos disponíveis nas bases de dados, bem como teses, dissertações, monografias, capítulos de livros eletrônicos e trabalhos completos publicados em anais de eventos disponibilizados gratuitamente em meio eletrônico. Não foi estipulado ano ou período de publicação das pesquisas, visando uma busca ampliada na literatura. Foram excluídos os artigos originais que não referenciaram no título, no resumo ou no texto o assunto abordado nesta revisão, bem como artigos de revisão da literatura e editoriais. 
Vale salientar que não foi realizado o cadastro na plataforma "PROSPERO", tendo em vista que o número de pesquisas que relacionam a prática da Terapia Ocupacional na Equoterapia é escasso. Desta forma, este estudo apresenta risco de utilização de textos antigos e, consequentemente, a existência de termos desatualizados.

A pesquisa bibliográfica foi realizada nas plataformas de busca PubMed e Bireme e nas bases de dados: Scientific Electronic Library Online (SciELO), Literatura LatinoAmericana e do Caribe em Ciências da Saúde (LILACS) e Medical Literature Analysis and Retrieval Sistem on-line (MEDLI ). Para a pesquisa nas bases de dados foram utilizadas as seguintes palavras-chaves entre descritores presentes nas bases DeCS e MeSH e termos livres (TL) (palavras não encontradas nas bases de descritores, mas importantes para a coleta dos textos) nos seus correspondentes em inglês: Hipoterapia (TL), Intervenção (TL), Terapia Assistida por Cavalos (DeCS/MeSH) e Terapia
Ocupacional (DeCS / MeSH). O termo livre Equoterapia foi pesquisado apenas no idioma português por não haver correspondente desta palavra na língua inglesa.

A busca foi realizada em meio on-line de forma independente por dois pesquisadores, seguindo os critérios de inclusão e exclusão, utilizando os seguintes cruzamentos em português e seus correspondentes na língua inglesa: Equoterapia and Terapia Ocupacional; Equoterapia and Intervenção; Hipoterapia and Terapia Ocupacional; Hipoterapia and Intervenção; Terapia assistida por cavalos and Terapia Ocupacional; Terapia assistida por cavalos and Intervenção (Tabela 1). Os cruzamentos realizados com o termo "prática profissional" foram excluídos por não apresentar nenhum material nas bases de dados pesquisadas. A coleta dos dados foi realizada nos meses de junho e julho de 2018, com data final de busca no dia 11 de julho de 2018.

TABELA 1

APRESENT ÇÃ̃o dos CRUZAMENTOS NAS BASES DE DADOS E OS RESUlTADO DE ARTIGOS ENCO RADOS.

\begin{tabular}{llll}
\hline Cruzamentos inseridos na revisão & $\begin{array}{l}\text { Total } \\
\text { encontrados }\end{array}$ & $\begin{array}{l}\text { Cruzamentos excluídos da } \\
\text { revisão }\end{array}$ & $\begin{array}{l}\text { Total } \\
\text { encontrados }\end{array}$ \\
\hline $\begin{array}{l}\text { Equine-assisted Therapy and } \\
\text { intervention. }\end{array}$ & 138 & $\begin{array}{l}\text { Equine-assisted Therapy and } \\
\text { Professional practice. }\end{array}$ & 0 \\
$\begin{array}{l}\text { Equine-assisted Therapy and } \\
\text { Occupational Therapy. }\end{array}$ & 17 & $\begin{array}{l}\text { Equoterapia and Prática } \\
\text { profissional. } \\
\text { Hippotherapy and Prática } \\
\text { profissional. }\end{array}$ & 0 \\
$\begin{array}{l}\text { Equoterapia and Intervenção. } \\
\text { Equoterapia and Terapia Ocupacional. }\end{array}$ & 70 & $\begin{array}{l}\text { Terassistida por cavalos } \\
\text { and Prática profissional. }\end{array}$ & 0 \\
$\begin{array}{l}\text { Hipoterapia and Intervenção. } \\
\text { Hipoterapia and Terapia Ocupacional. }\end{array}$ & 3 & & \\
$\begin{array}{l}\text { Terapia assistida por cavalos and } \\
\text { Intervenção. }\end{array}$ & 7 & \\
$\begin{array}{l}\text { Terapia assistida por cavalos and } \\
\text { Terapia Ocupacional. }\end{array}$ & 1 & & \\
\hline
\end{tabular}




\section{Extração dos dados}

Por meio da utilização dos descritores e termos livres definidos anteriormente, foi realizada a identificação e seleção dos artigos nas bases de dados escolhidas. Inicialmente foram lidos os títulos dos artigos e os selecionados pela relevância do título foram submetidos à leitura dos resumos e, se enquadrados nos critérios de inclusão preestabelecidos, analisados na íntegra, seguindo um protocolo criado para tal fim. Os artigos finalmente selecionados foram aqueles que atenderam a todos os critérios de elegibilidade expostos, que possibilitaram responder ao questionamento desta revisão e que foram avaliados com qualidade de acordo com o teste de relevância proposto no estudo de Azevedo
(2010). Este teste estabelece quatro questões para credibilidade da pesquisa que devem ser avaliadas com resultado positivo ou negativo (Quadro 1). Os dados dos artigos de interesse (que responderam positivamente as quatro perguntas do teste de relevância) foram detalhadamente analisados por meio de um fichamento criado para o presente estudo com as seguintes categorias: autores, ano/local, população, profissionais, intervenção e resultados.

Os resultados foram expostos através de um diagrama e em quadros com a análise das categorias elencadas no fichamento previamente criado com base nos critérios de seleção e objetivo deste estudo.

QUA

1

TESTE DE RELEVÂNCIA APLICADO NA ÍNTEGRA AOS ARTIGOS SELECIONADOS.

\begin{tabular}{|c|c|c|}
\hline Questões norteadoras & Sim & Não \\
\hline 1. O problema da pesquisa está claro? & & \\
\hline $\begin{array}{l}\text { 2. Os objetivos do estudo têm relação com a questão que está sendo } \\
\text { estudada? }\end{array}$ & & \\
\hline 3. A metodologia está descrita com clareza e alcança os objetivos? & & \\
\hline $\begin{array}{l}\text { 4. Os resultados são compatíveis com a metodologia e merecem } \\
\text { credibilidade? }\end{array}$ & & \\
\hline
\end{tabular}

O estudo foi incluso? Sim ( ) Não ( )²

\section{Resultados}

$\mathrm{A}^{3}$ partir da busca de descritores e termos livres foram identificados 194 artigos, sendo 155 na PubMed, 31 na Bireme 8 na SCIELO; Após a leitura dos títulos, conforme o critério de inclusão estabelecido, 68 estudos foram incluídos e selecionados para a leitura do resumo, e 37 para leitura na íntegra (Figura 1). Após aplicação de elegibilidade foi realizada a avaliação da qualidade dos artigos através de um protocolo (Quadro 1) baseado no estudo de Azevedo (2010), sobrando assim 3 artigos para análise nesse estudo (Tabela 2).

3 Nota. Fonte: Recuperado de "Sobrecarga do cuidador informal da pessoa idosa frágil: uma revisão sistemática," de R. S. Azevedo, 2010, p. 41. 
FIGUR 1

\section{NÚMER DE ARTIGOS ENCONTRADOS E SELECIONADOS DE ACORDO COM OS CRITÉRIOS DE ELEGIBILIDADE.}

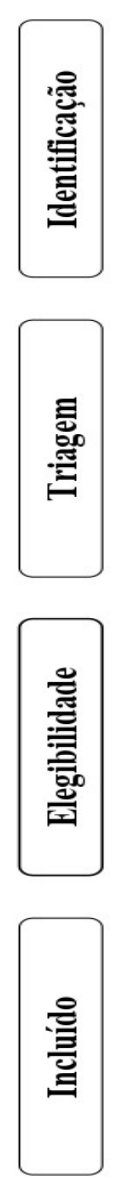

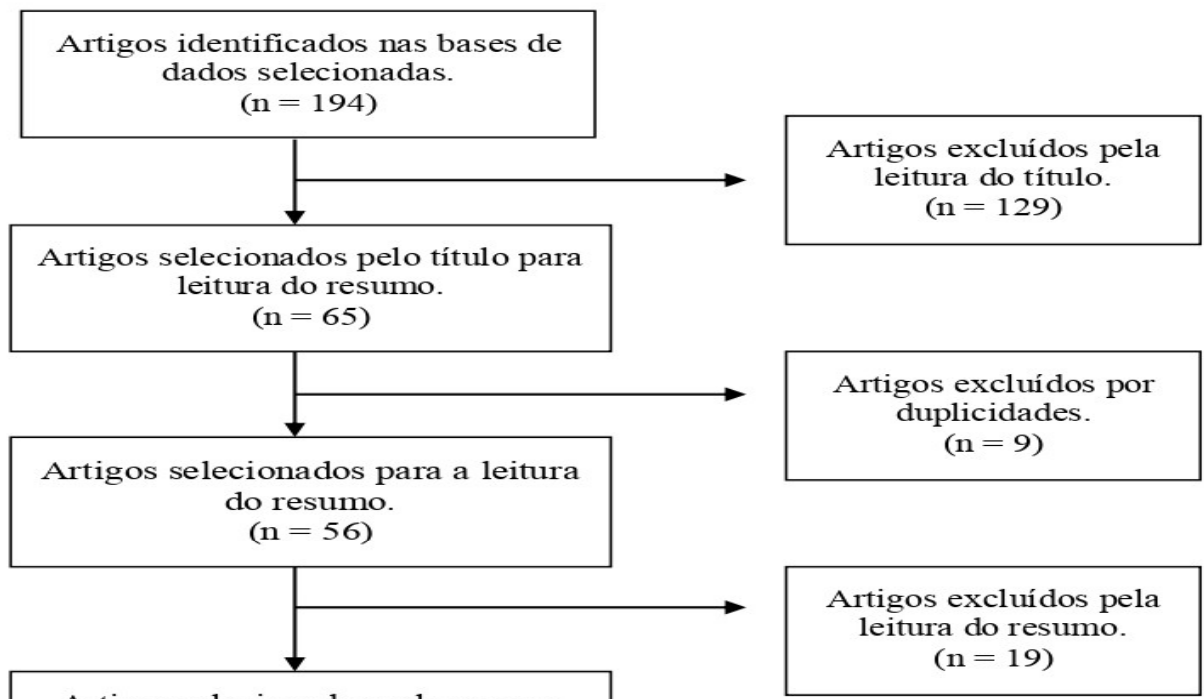

Artigos selecionados pelo resumo para leitura na íntegra. $(n=37)$

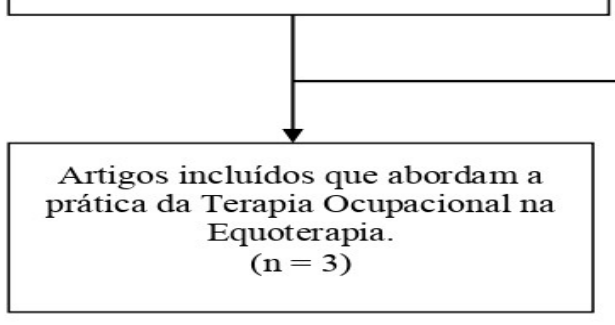

Artigos excluídos por:

não abordarem a prática da Terapia Ocupacional $(\mathrm{n}=30)$

realizarem apenas sessões de Terapia Ocupacional clínica antes ou durante a coleta de dados $(n=2)$

acesso público do artigo fechado $(n=2)$
Os artigos excluídos durante o processo de seleção (Figura 1), em conformidade com os critérios de exclusão estabelecidos, não apresentaram em seu título, resumo e na integra alguns dos quesitos como: relação com a prática da Terapia Ocupacional na Equoterapia, apresentavam-se em idiomas que diferiam do português e inglês, sua metodologia não estava clara, realizaram sua pesquisa com prancha simuladora e não com o cavalo e seu acesso era possível apenas mediante pagamento. Os artigos incluídos constavam em seu conteúdo a prática efetiva da Terapia Ocupacional na Equoterapia e contemplavam todos os quesitos descritos a cima, além de responderem positivamente as quatros questões do teste de relevância proposto no estudo de Azevedo (2010) (Quadro 1).
Para análise dos dados contidos nos artigos, a Tabela 2 apresenta as categorias estabelecidas no protocolo criado para a extração das principais informações dos estudos. Desta forma, foram analisados, o ano e local da publicação, a população que foi atendida na Equoterapia, os profissionais que participavam doa atendimentos, o tipo de intervenção realizada e os resultados obtidos através desta intervenção. 
TABELA 2

PRINCIPAI CARACTERÍSTICAS DOS ESTUDOS INCLUÍDOS NA REVISÃO.

\begin{tabular}{|c|c|c|c|c|c|}
\hline Autor & Ano/Local & População & Profissionais & Intervenção & Resultados \\
\hline $\begin{array}{l}\text { Champagne; } \\
\text { Corriveau \& } \\
\text { Dugas. }\end{array}$ & $\begin{array}{l}\text { 2016/ } \\
\text { Califórnia, San } \\
\text { Diego. }\end{array}$ & $\begin{array}{l}\text { Crianças com } \\
\text { Paralisia Cere- } \\
\text { bral, idade } \\
\text { entre } 4 \text { e } 16 \\
\text { anos. }\end{array}$ & $\begin{array}{l}1 \text { Terapeuta } \\
\text { Ocupacional, } \\
1 \text { condutor de } \\
\text { cavalos e } 2 \\
\text { acompanhan- } \\
\text { tes laterais. }\end{array}$ & $\begin{array}{l}30 \text { minutos de } \\
\text { sessão, uma } \\
\text { vez por semana, } \\
\text { durante } 10 \text { sema- } \\
\text { nas realizando a } \\
\text { preparação do } \\
\text { cavalo, montando } \\
\text { e desmontando, } \\
\text { o alimentando e } \\
\text { executando ativi- } \\
\text { dades com ele em } \\
\text { movimento. }\end{array}$ & $\begin{array}{l}\text { Melhora na pre- } \\
\text { cisão motora fina, } \\
\text { desenhar uma } \\
\text { linha ao longo } \\
\text { de um trajeto e } \\
\text { dobrar um papel, } \\
\text { e grossa. Aumento } \\
\text { da força muscular } \\
\text { do tronco. }\end{array}$ \\
\hline $\begin{array}{l}\text { Park; Rha; Shin; } \\
\text { Kim \& Jung. }\end{array}$ & $\begin{array}{l}2014 \text { / Seul, } \\
\text { Coréia do Sul. }\end{array}$ & $\begin{array}{l}\text { Crianças com } \\
\text { Paralisia Cere- } \\
\text { bral, idade } \\
\text { entre } 3 \text { e } 12 \\
\text { anos. }\end{array}$ & $\begin{array}{l}1 \text { Terapeuta } \\
\text { Ocupacional, } \\
1 \text { Assistente } \\
\text { treinado e } 1 \\
\text { acompanhante } \\
\text { lateral. }\end{array}$ & $\begin{array}{l}45 \text { minutos de } \\
\text { sessão, } 2 \text { vezes por } \\
\text { semana, durante } \\
8 \text { semanas reali- } \\
\text { zando atividades } \\
\text { voltadas para o } \\
\text { desenvolvimento } \\
\text { de habilidades } \\
\text { sensório-motoras } \\
\text { e perceptivo-mo- } \\
\text { toras. }\end{array}$ & $\begin{array}{l}\text { Melhora na função } \\
\text { motora grossa e } \\
\text { no desempenho } \\
\text { funcional no auto- } \\
\text { cuidado, na mobi- } \\
\text { lidade e funciona- } \\
\text { mento social. }\end{array}$ \\
\hline $\begin{array}{l}\text { Gabriels; Pan; } \\
\text { Dechant; } \\
\text { Agnew; Brim \& } \\
\text { Mesibov }\end{array}$ & 2015 & $\begin{array}{l}\text { Crianças e ado- } \\
\text { lescentes, entre } \\
6 \text { e } 16 \text { anos, } \\
\text { com Autismo. }\end{array}$ & $\begin{array}{l}1 \text { instrutor de } \\
\text { equitação } \\
\text { terapêutica e } 2 \\
\text { acompanhan- } \\
\text { tes laterais. }\end{array}$ & $\begin{array}{l}45 \text { minutos de } \\
\text { sessão, duran- } \\
\text { te } 10 \text { semanas } \\
\text { realizando uma } \\
\text { rotina consistente: } \\
\text { colocar capacete, } \\
\text { esperar no banco, } \\
\text { montaria, ativida- } \\
\text { des de equitação, } \\
\text { desmonte, lim- } \\
\text { peza e guardar } \\
\text { equipamentos. }\end{array}$ & $\begin{array}{l}\text { Benefícios na } \\
\text { autorregulação, } \\
\text { socialização, } \\
\text { comunicação, } \\
\text { comportamentos } \\
\text { adaptativos e } \\
\text { motores. }\end{array}$ \\
\hline
\end{tabular}




\section{Dıscussão}

É perceptível o aumento do número de estudos que apresentam os efeitos da Equoterapia no tratamento de seus praticantes acometidos por múltiplas patologias (Salineiro, Arruda, \& Alves, 2002; Park, Rha, Shin, Kim, \& Jung, 2014; Gabriels et al., 2015; Bender \& Guarany, 2016; Champagne; Corriveau \& Dugas, 2016; Koca, \& Ataseven, 2015), entretanto, é notório a incipiência de trabalhos que exponham os benefícios da prática da Terapia Ocupacional atrelado a esse método terapêutico, como foi possível observar nos resultados do presente estudo, o qual se propôs a identificar e discorrer sobre pesquisas que abordem a intervenção do Terapeuta Ocupacional na Equoterapia (Champagne et al., 2016; Park et al. (2014); Gabriels et al., 2015) .

Na tabela 1, é possível observar que as publicações dos estudos selecionados ocorreram nos últimos 5 anos, deixando a entender que se trata de uma prática nova. Entretanto, os benefícios da utilização do cavalo em processos terapêuticos são relatados desde a Antiguidade. Botelho (1997) apresenta que já é possível encontrar referência acerca da equitação no compêndio das Dietas de Hipócrates De Loo (458-377 a.C.), a qual ele recomendava sua prática para reabilitar a saúde, preservar o corpo humano de doenças e proporcionar a melhora do tônus muscular. Igualmente, em "A Arte Ginástica" de Merkurialis, o qual relata que a equitação fornece contribuições tanto para o corpo como para os sentidos do praticante.

O prelúdio da Terapia Ocupacional também não é recente, de acordo com Moreira (2008), no início do século XX, surgiu nos EUA formalmente como profissão. Nesse período, em consequência da I e II Guerra Mundial, atuava com o escopo de minimizar os impactos físicos e mentais sofridos pelos soldados que estavam em serviço. Em contrapartida, o Conselho Regional de Fisioterapia e Terapia Ocupacional da $9^{\circ}$ região que corresponde aos Estados de Mato Grosso, Acre e Rondônia (CREFITO 9) (n.d.) apresenta o início do movimento dessa profissão no Brasil como forma de tratamento para indivíduos com doenças crônicas, como: tuberculose, acidentes de trabalho, doenças ocupacionais, entre outros. No entanto, a Equoterapia só foi reconhecida pelo Conselho Federal de Fisioterapia e Terapia Ocupacional (COFFITO) como recurso e componente constituinte da formação da Terapia Ocupacional em 2008, por meio da Resolução $N^{\circ}$. 348. de 27 de março de 2008.
Identificamos durante o levantamento que a maioria das publicações encontradas são artigos realizados em territórios internacionais, no entanto o processo de conhecimento e valorização da Equoterapia no Brasil já havia iniciado desde 1989, através das atividades equestres realizadas pela ANDE e foi reconhecida pelo Conselho Federal de Medicina, como prática clínica, em 1997 (Associação Brasileira de Equoterapia [ANDE], n.d.c). Também é importante elucidar que existe no Brasil a Revista Brasileira de Equoterapia (RBE), órgão oficial da ANDE-BRASIL e um meio de comunicação específico e responsável pela divulgação de trabalhos científicos voltados para essa temática e suas áreas de competência.

Esse conjunto de circunstâncias apresentadas enfatizam os questionamentos acerca da escassez de publicações por parte dos profissionais intrínsecos ao método, tendo em vista que as pesquisas são uma ferramenta importante para transmitir e adquirir novos conhecimentos. Assim como afirma o Fórum Global de Pesquisa em Saúde (2007), ao discorrer que são necessárias novas informações e a criação de métodos mais eficazes e com maior potencialidade para garantir a proteção e promoção da saúde, uma vez que estamos sempre em evolução e, consequentemente, alguns conhecimentos e as estratégias já existentes nem sempre são efetivas na resolução de atuais problemas de saúde.

O Fórum de Reflexão Universitária (2002) revela que mesmo que a pesquisa científica no Brasil tenha progredido significativamente dentre os últimos quarenta anos, essa prática ainda é mal distribuída, de modo que a maioria dos indivíduos se tornam dependentes dos pesquisadores e dos estudos mais influentes. E o obstáculo encontrado para a quebra desse ciclo vicioso e para a criação de uma cultura científica, o qual exige inúmeros investimentos em educação e cultura, é a ausência de um sólido sistema de educação pública que incentive a pesquisa, assim como a falta de financiamento.

Dentre os estudos elegidos, verificou-se que o público atendido foi o infantojuvenil, com faixa etária entre 3 e 16 anos (Champagne et al., 2016; Park et al., 2014; Gabriels et al., 2015). Apesar dos dados apresentados, não foram encontradas evidências científicas que comprovem uma maior eficácia do método com essa população, assim como não foi evidenciado se os pais e responsáveis recorrem ao tratamento precocemente. É lúcido que durante a infância o processo de desenvolvimento humano está sujeito a diversas alterações no 
aspecto motor, cognitivo, afetivo e social, resultantes do processo de amadurecimento do sistema nervoso central, obtendo uma grande influência de estímulos consequentes da interação dos fatores ambientais e biológicos. Do mesmo modo, a fase da adolescência também é marcada por múltiplas alterações biológicas, físicas e funcionais, as quais estão associadas ao pico de produção de hormônios de ambos os sexos (Ré, 2011).

Utilizando as palavras de P. F. Santos (2012), reafirmamos que as habilidades motoras e cognitivas estão em constante evolução durante o período referido, e a implementação da Equoterapia se faz importante, tendo em vista que tal prática é apontada como responsável por propiciar estímulos corporais para a criança durante o trajeto realizado em cima do cavalo, auxiliando no desenvolvimento psicomotor e proporcionando ao praticante maior possibilidade de controlar seu próprio corpo.

Tomado conhecimento de tais circunstâncias, R.B. Santos, Cyrillo, Sakakura, Perdigão e Torriani (2006) salientam que é indispensável o processo de escolha do cavalo adequado para cada praticante, visto que o nível de tônus muscular do indivíduo influi diretamente na seleção da frequência do passo do animal. Responsável por proporcionar os distintos estímulos ao corpo, o autor também aponta que o animal mais indicado para praticantes hipotônicos é aquele que antepista, pois ao realizar o maior número de passadas por minuto os receptores proprioceptivos e articulares são ativados, os quais oportunizam o aumento do tônus muscular. Já para praticantes hipertônicos é recomendado o uso do cavalo que apresente uma frequência de passo mais lenta, transpistar, por ocasionar um declínio nos estímulos proprioceptivos e no tônus muscular, uma vez que o sistema vestibular é acionado lentamente.

As vantagens ultrapassam os fatores físicos, também é possível observar contribuições nas relações afetivas e sociais dos indivíduos. De acordo com Freire e Potsch (2009), a interação entre o praticante e o cavalo durante as sessões viabiliza a busca por comunicação e socialização ao demonstrar sentimentos e manifestações que expandem sua capacidade cognitiva.

São notórios os benefícios propiciados pelo método, porém são existentes contraindicações para sua aplicação. Mediante a realização de uma pré-avaliação a fim de verificar se a prática é a mais apropriada para os indivíduos mediante suas potencialidades apresentadas, Medeiros e Dias (2008) descrevem com clareza algumas contraindicações que perpassam desde aspectos cognitivos até os físicos, sendo elas: fobia ao animal, excessiva ansiedade, nível cognitivo baixo, luxação e subluxação de quadril, artrose de quadril, instabilidade atlanto-occipital, fibromialgia, convulsões, hipertensão descontrolada e ferimentos abertos.

As intervenções equoterápicas devem ser realizadas por uma equipe base composta por um mediador, auxiliar lateral e auxiliar guia que trabalharão em conjunto com o cavalo (Pereira, Lopes \& Figueiredo, 2015). De acordo com o Conselho Regional de Fisioterapia e Terapia Ocupacional da $15^{\text {a }}$ região que corresponde ao Estado do Espírito Santo (CREFI 15) (n.d.), disposto de um pilar multiprofissional e interdisciplinar, os mediadores capacitados devem ser atuantes no campo da saúde, educação e equitação, tais como: assistente social, fisioterapeuta, fonoaudiólogo, pedagogo, educador físico, psicólogo, terapeuta ocupacional e outros.

Tendo conhecimento de que a Terapia Ocupacional também está apta para atuar em tal campo, Koca \& Ataseven (2015) discorrem os objetivos da referida profissão na Equoterapia ao relatarem que os movimentos do cavalo são utilizados com a intenção de promover ganhos motores, cognitivos e no processamento sensorial, de modo a trabalhar em conjunto com os sistemas sensoriais e, consequentemente, obter melhora no desempenho das Atividades de Vida Diárias (AVD). Além das AVD, o terapeuta ocupacional está capacitado para desenvolver atividades que contemple todas as outras ocupações humanas. $\mathrm{O}$ atendimento estará sendo planejado de acordo com a avaliação inicial e com as demandas identificadas durante o processo e, também, das queixas apresentadas pelo próprio praticante, seus genitores ou responsáveis.

Na tabela 1, o terapeuta ocupacional é o profissional responsável pelos atendimentos durante as coletas de dados, apesar de não ser evidenciado como mediador no estudo de Gabriels et al. (2015), ele se faz presente na aplicação e administração do Teste de proficiência motora de Bruininks-Oseretsky e de duas subescalas do Teste de Integração Sensorial e Praxis com os praticantes selecionados. Suas contribuições na Equoterapia transpõe a capacidade de aplicar testes, como consta nos 
outros artigos selecionados (Champagne et al., 2016; Park et al., 2014), nos quais o terapeuta ocupacional conduziu as intervenções equoterápicas.

As sessões ocorreram em arenas internas, externas e/ou trilhas. Os praticantes desempenharam funções singulares, como equipar o cavalo para as sessões, alimentá-lo, realizar montaria e desmontaria e guardar os equipamentos após o término das sessões.

Ainda discutindo a tabela 1, os dados mostram que o tempo preeminente de duração das intervenções foi de 45 minutos, durante 10 semanas, ocorrendo aproximadamente uma vez na semana. Não foram localizados registros na literatura que estabeleçam um tempo padrão para as sessões, uma vez que cada praticante é detentor de demandas específicas e individuais. Assim como Thiel (2006) apresenta em seu relato de experiência com um cliente indicado por um terapeuta ocupacional que necessitava de uma terapia com curta duração, os resultados comprovaram que poucas sessões também geram resultados positivos e estão inclinadas a atingir os objetivos estabelecidos previamente. Em conformidade Galvão; Sutani, Pires, Prada e Cordeiro (2010), relata que seu estudo foi composto por 10 sessões, entretanto mesmo com a mostra de resultados favoráveis é requerido que ocorra mais sessões com o intuito de comprovar sua eficácia.

As possibilidades de intervenção da Terapia Ocupacional na Equoterapia são bastante vastas. Como já abordado anteriormente, o terapeuta poderá intervir com enfoque nas AVD do praticante. Empregando as palavras de Salineiro, Arruda e Alves (2002), as atividades de vida diária poderão ser atreladas aos cuidados demandados do cavalo, tais como: banho, alimentação e vestuário adequado (equipamentos). O cliente estará desenvolvendo essas práticas com o animal a fim de propiciar uma melhor compreensão dessas atividades dentro das suas vivências como ser humano, igualmente da importância delas.

Observando a Tabela I e dando continuidade na identificação dos ganhos obtidos através da atuação do terapeuta ocupacional na Equoterapia, Gabriels et al. (2015) apresentam em seu estudo o resultado da comparação de dois grupos denominados pelos autores como grupo THR, que participou de atividades equoterápicas, e o grupo BA, que não obteve interação com o cavalo, e concluíram que o THR apresentou melhoras significativas em aspectos da cognição social, comunicação, sociabilidade e diminuição da irritabilidade e hiperatividade. Assim como consta no estudo de Salineiro, Arruda e Alves (2002), ao afirmar que a Equoterapia é um método de tratamento que também traz benefícios aos usuários dos serviços da saúde mental, como o esquizofrênico. O texto apresenta que as atividades, para essa população, foram elaboradas e executadas com o objetivo principal de restituir a saúde e prevenir futuros surtos de tais indivíduos. Os ganhos proporcionados pela junção da Terapia Ocupacional e da Equoterapia alcançaram o desenvolvimento e a melhora na sociabilidade, aspectos cognitivos, diminuição da ansiedade e elevação da autoestima.

Já na pesquisa de Park et al. (2014), os seus resultados mostraram que a Equoterapia aumentou o desempenho funcional de pacientes com Paralisia Cerebral (PC) e proporcionou ganhos na função motora grossa, os quais foram mensurados através do GMFM-88, GMFM-66, escalas que são utilizadas para medir mudanças na função motora grossa, e o PEDI, Inventário de Avaliação Pediátrica de Incapacidade. Os achados na pesquisa de Champagne, Corriveau e Dugas (2016), também discorrem sobre os benefícios motores conquistados em crianças com PC nos níveis I e II através do GMFCS, Sistema de Classificação da Função Motora Grossa, o qual permitiu observar melhora nas funções motoras grossas: ficar em pé, andar, correr, pular e nas habilidades motoras quanto ao equilíbrio, força, precisão e motricidade fina. Da mesma forma, Bender \& Guarany (2016) ao comparar os grupos GP, praticante da Equoterapia, e GPN, não praticantes, revelam por intermédio do teste PEDI que a área de mobilidade apresentou maiores pontuações para o GP.

Evidentemente, os resultados apresentados e discutidos na presente revisão de literatura nos outorgam a afirmar que a interação entre homem e cavalo, proporcionado pela Equoterapia, é detentora de inúmeros benefícios, bem como a atuação do Terapeuta Ocupacional mostrou-se ser eficaz. No entanto, embora posto que os resultados terapêuticos sejam satisfatórios, ainda se faz necessário mais estudos na área. Tendo em vista que o trabalho em conjunto com o cavalo proporciona ganhos que transcendem os aspectos motores e cognitivos, aqui recomendamos que pesquisas de intervenção na área sejam traçadas e investigadas para contribuir com 
o desenvolvimento integral do sujeito e para expandir as possibilidades de prática do terapeuta ocupacional, bem como tornar de conhecimento geral seus feitos e salientar a necessidade da implantação de conteúdo ou disciplinas nas grades curriculares que ofereçam suporte para a criação de projetos que produzam conhecimento prático e científico sobre tal método terapêutico.

Durante o percurso da realização da presente revisão integrativa foram observadas algumas limitações, tais como: incipiência de pesquisas na área, não contemplação de outros idiomas além do português e do inglês e não contar com financiamento para custear a pesquisa. Desta forma, sugerimos estudos que contemplem as limitações aqui destacadas. Além disso, sugerimos publicações de relatos de experiências para que o reconhecimento dos profissionais, da sua atuação e dos resultados obtidos através do uso da Equoterapia sejam comprovados.

\section{RefERÊNCIAS BIBLIOGRÁFICAS}

Associação Americana de Terapia Ocupacional. (2015). Estrutura da prática da Terapia Ocupacional: Domínio \& Processo (3 a ed., 26). (Cavalcanti, A., Dutra, F. C. M.S., \& Elui, V. M. C., Trad.). Revista de Terapia Ocupacional da Universidade de São Paulo, pp.1-49, Quadrimestral. São Paulo, Brasil. (Obra original publicada em 2014). Recuperado em: http://www.revistas.usp.br/rto/article/ view/97496/96423. doi: http://dx.doi.org/10.11606/issn.22386149.v26iespp1-49

Associação Nacional de Equoterapia (ANDE-BRASIL). (n.d.a). O Método. Recuperado de http://equoterapia.org.br/articles/index/ article_detail/142/2022

Associação Nacional de Equoterapia (ANDE-BRASIL). (n.d.c). Reconhecimento. Recuperado de http://equoterapia.org.br/articles/ index/article_detail/140/2024

Associação Nacional de Equoterapia (ANDE-BRASIL). (n.d.b). Quem somos: Certificação. Recuperado em: http://equoterapia.org. br/articles/index/articles_list/134/80/0

Azevedo, R. S. (2010). Sobrecarga do cuidador informal da pessoa idosa frágil: uma revisão sistemática (Dissertação de Mestrado). Curso de Enfermagem, Universidade Federal de Minas Gerais, Belo Horizonte, Brasil. Recuperado em http://www.bibliotecadigital.ufmg.br/dspace/bitstream/handle/1843/GCPA-8D9LAL/ raquel_souza_azevedo.pdf?sequence $=1$

Bender, D., \& Guarany, N. (2016). Efeito da equoterapia no desempenho funcional de crianças e adolescentes com autismo. Revista de Terapia Ocupacional da Universidade de São Paulo, 27(3), pp. 271-277. doi:https://doi.org/10.11606/issn.2238-6149.v27i3p271-277
Botelho, L. A. A. (1997). A hipoterapia na medicina de reabilitação. Acta Fisiatrica, 4(1), 44-46, Trimestral. Recuperado de http:// www.revistas.usp.br/actafisiatrica/article/view/102065/100484

Cavalcanti, V. A. S., \& Calil, F. C. (2007). Equoterapia. In: A. Cavalcanti, \& C. R. C., Galvão. Terapia Ocupacional: Fundamentação e Prática. (cap. 53, pp. 494-501). Rio de Janeiro: Guanabara Koogan.

Citterio, D. (1999). A hipoterapia na recuperação da pessoa portadora de deficiência e as atividades pré-esportivas. Anais do Congresso Brasileiro de Equoterapia, São Paulo, SP, Brasil.

Conselho Regional de Fisioterapia e Terapia Ocupacional da $3^{\circ}$ Região. (n.d.) Cartilha: Terapeuta Ocupacional e o SUS. Bela Vista, São Paulo, Brasil. Recuperado em http://www.crefito3.org.br/dsn/ pdfs/Cartilha\%20-\%20t.o.pdf

Conselho Regional de Fisioterapia e Terapia Ocupacional da $9^{\circ}$ Região. (n.d.). 100 de Terapia Ocupacional no mundo. Cuiabá, Mato Grosso, Brasil. Recuperado de http://www.crefito9.org.br/ terapia-ocupacional/o-que-e-terapia-ocupacional/noticias/ periodo-para-renovacao-de-drf-e-de-1-a-30-de-abril-de-2017/ noticias/100-anos-da-terapia-ocupacional-no-mundo/1163

Conselho Regional de Fisioterapia e Terapia Ocupacional da $15^{\circ}$ Região. (n.d.) Equoterapia: fonte de reabilitação global do indivíduo com o suporte psicológico e motor sobre o cavalo. Vitória, Espírito Santo, Brasil. Recuperado de http://www.crefito15.org.br/ equoterapia-fonte-de-reabilitacao-global-do-individuo-como-suporte-psicologico-e-motor-sobre-o-cavalo/

Champagne, D., Corriveau, H., \& Dugas, C. (2016). Effect of Hippotherapy on Motor Proficiency and Function in Children with Cerebral Palsy Who Walk. Physical \& Occupational Therapy in Pediatrics, 37(1), 51-63.

Fontana, R. et al. (2010). Therapeutic process and perspectives about the practice of equine-assisted therapy. Revista de Enfermagem Ufpe On Line, [s.I.], 4(2), 757-763.

Fórum Global de Pesquisa em Saúde. (2007). Por que pesquisa em saúde? / Ministério da Saúde, Secretaria de Ciência, Tecnologia e Insumos Estratégicos \& Departamento de Ciência e Tecnologia. - Brasília: Ministério da Saúde. (Série B. Textos Básicos de Saúde), (Série Pesquisa para Saúde: Textos para Tomada de Decisão).

Fórum de Reflexão Universitária. (2002). Desafios da pesquisa no Brasil: uma contribuição ao debate, 16(4), 15-23. São Paulo em Perspectiva. Recuperado em http://www.scielo.br/scielo. php?script=sci_arttext\&pid=S0102-88392002000400004. doi: http://dx.doi.org/10.1590/S0102-88392002000400004

Freire, H. B. G. (1999) Equoterapia teoria e técnica: uma experiência com crianças autistas. São Paulo: Vetor.

Freire, H. B. G., \& Potsch, R. R. (2009). O autista na equoterapia: a descoberta do Cavalo. Brasil. Recuperado em: http://www.universoautista.com.br/autismo/modules/news/article.php?storyid=476

Gabriels, R. L., Pan, Z., Dechant, B., Agnew, J. A., Brim, N., \& Mesibov, G. (2015). Randomized controlled trial of therapeutic horseback riding in children and adolescents with autism spectrum disorder. 
Journal of the American Academy of Child \& Adolescent Psychiatry, 54(7), 41-549.

Gadelha, M. N. (2016) Terapia ocupacional, equoterapia e síndrome de down: possibilidades de uma intervenção. (Monografia de Graduação) - Curso de Terapia Ocupacional, Centro de Ciências da Saúde, Universidade Federal da Paraíba, João Pessoa, Brasil.

Galvão, A., Sutani, J., Pires, M. A., Prada, S. H., Cordeiro, T. L. (2010). Estudo de Caso: A equoterapia no tratamento de um paciente adulto portador de ataxia cerebelar. Revista Neurociências, 18(3), 353-358. Recuperado em: http://www.revistaneurociencias.com. br/edicoes/2010/RN1803/407\%20relato\%20de\%20caso.pdf

Koca, T. T., \& Ataseven, H. (2015). What is hippotherapy? The indications and effectiveness of hippotherapy. Northern Clinics of Istanbul, 2(3), 247-252, Trimestral. Kare Publishing. Recuperado em: https://www.ncbi.nlm.nih.gov/pmc/articles/PMC5175116/ pdf/NCl-2-247.pdf

Luz, M. L. S., Boaretto, M. D., \& Rodrigues, J. M. Z. (2018). O trabalho em um Centro de Equoterapia sob a compreensão da Ergonomia da atividade. Editora Poisson, 21(1), 134-144. Belo Horizonte, Brasil. Recuperado em: http://www.abepro.org.br/biblioteca/ TN_STO_241_397_34518.pdf

Medeiros, M., Dias, E. (2008). Equoterapia: noções elementares e aspectos neurocientíficos. Rio de Janeiro: Revinter.

Mendes, K. S., Silveira, R. C. C. P., \& Galvão, C. M. (2008). Revisão integrativa: método de pesquisa para a incorporação de evidências na saúde e na enfermagem. Texto Contexto-Enferm, 17(4), 758-764. Florianópolis, Santa Catarina, Brasil.

Moreira, A. B. (2008). Terapia ocupacional: história crítica e abordagens territoriais/comunitárias. Revista Vita et Sanitas, 2(2), 79-90. Trindade, Brasil. Recuperado em: http://www.fug.edu.br/2018/ revista/index.php/VitaetSanitas/article/view/103/86

Park, E. S., Rha, D.-W., Shin, J. S., Kim, S., \& Jung, S. (2014). Effects of Hippotherapy on Gross Motor Function and Functional Performance of Children with Cerebral Palsy. Yonsei Medical Journal, 55(6), 1736-1742. Yonsei University College of Medicine, Seul, Coréia do Sul. Recuperado em: https://www.ncbi.nlm.nih.gov/ pmc/articles/PMC4205717/

Pereira, B. N., Lopes, G. C., \& Figueiredo, J. B. (2015). Contribuições da Equoterapia para o processo de ensino e aprendizagem dos praticantes com síndrome de Asperger. Anais do XII Congresso Nacional de Educação, 14638-14650. Curitiba, Paraná, Brasil. Recuperado em: https://docplayer.com.br/74770930-Contri buicoes-da-equoterapia-para-o-processo-de-ensino-e-apren dizagem-dos-praticantes-com-sindrome-de-asperger.html

Prestes, D. B., Weiss, S., \& Araújo, J. C. O. (2010). A equoterapia no desenvolvimento motor e autopercepção de escolares com dificuldade de aprendizagem. Revista Ciência e Cognição, 15(3), 192-203. Recuperado em: http://www.cienciasecognicao.org/ revista/index.php/cec/article/view/423/243
Ré, A. H. N. (2011). Crescimento, maturação e desenvolvimento na infância e adolescência: Implicações para o esporte. Motricidade, 7(3), 55-67. Recuperado em: http://www.scielo.mec.pt/pdf/ mot/v7n3/v7n3a08.pdf

Resolução n. 348. de 27 demarço de 2008. Dispõe sobre o reconhecimento, pelo Conselho Federal de Fisioterapia e Terapia Ocupacional, da Equoterapia como recurso terapêutico da Fisioterapia e da Terapia Ocupacional e dá outras providências. Recuperado de https://www.coffito.gov.br/nsite/?p=3110

Salineiro, A. L. S., Arruda, J. B. O., \& Alves, A. M. O. (2002). A contribuição da terapia ocupacional e equoterapia na reabilitação do esquizofrênico em fase inicial. Multitemas, (25), 76-91. Brasil. Recuperado em: http://www.multitemas.ucdb.br/article/view/842/817

Santos, P. (2012). Educação Não Formal e Equoterapia: O galope do educador na arena da terapia. (Dissertação de Mestrado) - Curso de Pedagogia, Centro Universitário Salesiano de São Paulo, Americana, Brasil. Recuperado em:https://unisal.br/wp-content/ uploads/2013/03/Disserta\%C3\%A7\%C3\%A3o_-Priscila-FernandaBertola-dos-Santos2.pdf

Santos, R. B., Cyrillo F. N., Sakakura M.T., Perdigão A.P., \& Torriani C. (2006). A Influência da Postura sobre o Cavalo e a Velocidade do Passo na Ativação dos Músculos Eretores Lombares através da Eletromiografia de Superfície. Colêtania de Trabalhos. XII Congresso Internacional de Equoterapia, Brasília. Conhecimentos Básicos sobre Equoterapia Versão CD-ROM.

Silveira, M. M., \& Wilbelinger, L.M. (2010). A equoterapia como recurso terapêutico no equilíbrio do idoso. Revista Brasileira de Ciências do Envelhecimento Humano, 7(1), 144-153. Editora: UPF. Recuperado em: http://seer.upf.br/index.php/rbceh/article/ view/563/930. doi:10.5335/rbceh.2010.014

Souza, M. T., Silva, M. D., \& Carvalho, R. (2010). Revisão integrativa: o que é e como fazer? O Einstein, 8(1), 102-106, Trimestral. São Paulo, Brasil. Recuperado em: http://www.scielo.br/pdf/eins/ v8n1/pt_1679-4508-eins-8-1-0102.pdf

Thiel, U. (2006). "Reparando" a personalidade ou dando apoios para o cotidiano. Epmt - equitação psicomotoraterapêutica. Anais do XII Congresso Brasileiro de Equoterapia, 70-83, Livros Completos. Brasília, Brasil.

Walter, G. B., \& Vendramini, O. M. (2000). Equoterapia: terapia como uso do cavalo. Minas Gerais: CPT/CEE-UFV. 